\title{
Investigating Behaviour and Face Encoding in a Hypothetical Real-World Social Contract: Handwashing in Hazardous Health Settings
}

\author{
Fatima M. Felisberti ${ }^{1}$ - Daniel Farrelly ${ }^{2}$
}

Published online: 5 May 2016

(C) The Author(s) 2016. This article is published with open access at Springerlink.com

\begin{abstract}
Investigations into the evolutionary origins of human cognition has shown that individuals' memory for others is influenced by the latter's behaviour in social contracts. Such research is primarily based on hypothetical or more abstract forms of social contracts, whereas an application of this knowledge to everyday health behaviours can be of great value. To address this, the current study investigated whether participants who were asked to imagine themselves in a hypothetical hazardous health scenario showed differential response sensitivity $\left(d^{\prime}\right)$ and latency (RT) to faces of hospital staff tagged with contrasting hand hygiene before touching patients: clean hands, dirty hands, or unknown handwashing behaviour (control). The test used a two-alternative forced-choice (2AFC: "old/new") face recognition paradigm. The findings showed that $d^{\prime}$ to dirty and clean hands were similar, but higher than for controls. Moreover, $d^{\prime}$ was not affected by the occupation of hospital staff (nurses vs. porters). The absence of memory gains towards clean or dirty hands points to the need for new strategies to remind patients to observe (and remember) the hand hygiene of others when exposed to hazardous health environments.
\end{abstract}

Keywords Hand hygiene $\cdot$ Cheater detection $\cdot$ Face recognition $\cdot$ Healthcare $\cdot$ Memory

Fatima M. Felisberti

f.felisberti@kingston.ac.uk

1 Psychology Department, Kingston University, Penrhyn Road, London KT1 2EE, UK

2 Institute of Health and Society, University of Worcester, Henwick Grove, Worcester WR2 6AJ, UK
The role of social exchange on the evolution of human cognition was explored by Cosmides and Tooby (1992), and as such is seen by many as being central to the founding principles of evolutionary psychology. They point to the evolutionary benefits of engaging in social contracts with others, and consequently the adaptations evident in our cognitive processes that ensure their successful running. Primarily, Cosmides and Tooby (1992) suggest that an ability to detect cheats in social contracts is an evolved mental module, and they famously point to heightened performance on the Wason selection task (Wason 1966) for social contract versions over abstract versions as evidence of this (Cosmides 1989). This effect was also found for unfamiliar social contracts (Cosmides 1989), and has since been observed crossculturally (Sugiyama et al. 2002). Furthermore the difference in cognitive processing of social contract over abstract tasks is further supported by the differences in performance of patients with brain damage (Stone et al. 2002) as well as the finding that emotional processing predicted reasoning on social contract tasks but not abstract ones.

As well as influencing reasoning, there is a great deal of evidence that individuals have a heightened recognition of others in social exchange scenarios. In particular, it has been shown that we have an enhanced memory and recognition for the faces of cheaters (Farrelly and Turnbull 2008; Mealey et al. 1996; Oda and Nakajima 2010; Oda 1997). However this is not always the case (e.g. Barclay and Lalumière 2006; Felisberti and Pavey 2010), and more recent research points to a more generally adaptive cognitive process whereby individuals displaying the rarer (Barclay 2008; Volstorf et al. 2011) or unexpected behaviour (Bell et al. 2012; Chang and Sanfey 2009; Kroneisen and Bell 2012; Suzuki and Suga 2010) are actually recalled better. Regardless whether the precise mechanism is an enhanced memory for cheaters only or an enhanced memory for less frequent behaviours (which often will 
be cheating), it is clear from the evidence that humans have an adaptive attention and memory for individuals in social exchanges (Bell and Buchner 2012).

The research into social exchanges has used a diverse range of scenarios to explore this. This includes every day examples such as tipping in restaurants (e.g. Farrelly and Turnbull 2008), artificial economic games such as the Prisoner's Dilemma (Volstorf et al. 2011) or hypothetical social contracts such as "If a man eats cassava root, then he must have a tattoo on his face" (Cosmides and Tooby 1992). However what is currently missing is an investigation of more applied social contracts, where certain social exchanges can be very important. For example, certain social exchanges can greatly influence an individual's health or wellbeing, so an understanding of reasoning and cognition here can be very informative and be used as the basis of real world interventions that can benefit the general public.

The aim of the current studies, therefore, is to investigate the applied social contract of hand-washing in health-care settings. When it comes to health care associated infections, the World Health Organization considers hand-washing the most important element for health care workers (HCWs) to attend to, to prevent their further spread (WHO 2009). Therefore, hand-washing is essential in the battle against crosstransmission of infectious microorganisms in high-risk environments. In response, a number of initiatives have been developed to increase hand hygiene amongst HCWs, such as the National Patient Safety Agency campaign entitled 'clean your hands', launched in the UK in 2004 (now defunct). Such initiatives can help greatly when properly executed, as a decrease in contamination when medical staff wore gloves or hand antisepsis during patient care shows (Pittet et al. 1999). However, despite the clear benefits of good hand hygiene the actual levels of compliance amongst HCWs can be quite low, often below $10 \%$ (Longtin et al. 2011; Sladek et al. 2008) showing that much is needed to be done to improve HCW compliance with good hand hygiene (Salmon et al. 2015).

Recently, attention has turned from targeting HCWs to the patients' role in encouraging and promoting good hand hygiene amongst different HCWs. A systematic review examined previous research on the effectiveness of different strategies to encourage patient participation in supporting hand hygiene amongst HCWs (Davis et al. 2015). It found that most studies focused on patients' intentions rather than their actual behaviour when it came to reminding HCWs to wash their hands. It concluded that future research would benefit from using more objective measures of patient behaviour, since only one study observed whether patients actually reminded HCWs of hand hygiene (Lent et al. 2009).

The willingness and confidence to question hand-washing behaviour may well rest on patients' memory of those HCWs that adhere to the protocol and those that do not. Therefore, it is our contention that hand-washing can be viewed as a social contract between HCWs and patients, whereby HCWs must wash hands before executing their tasks (cost) in the hospital environment before receiving their wages (benefit), in line with the social contract theory (Cosmides and Tooby 1992). However, due to the hazardous nature of potential outcomes, it is possible that attention to additional violations may play a role, such as those in Hazard Management rules. These involve scenarios where behaviour can result in hazardous outcomes, so that risk is reduced (e.g. if you enter a building site, you must wear a hard-hat) (Fiddick et al. 2000). Research has shown that reasoning about these rules activates different parts of the brain (Ermer et al. 2006; Fiddick et al. 2005; Stone et al. 2002) and violations evoke different emotions (Fiddick 2004) than with social contract rules. Farrelly and Turnbull (2008) found, however, that individuals who violated both types of rules were remembered better than those that adhered to them. From this they conclude that although different cognitive processes may be involved in the two types of rules, an overall "look for rule violators" algorithm is observed in how such processes affect face recognition behaviour (Farrelly and Turnbull 2008). This would be adaptive, as it is useful to remember individuals whose behaviour may put you in jeopardy as well as being cheated on. This will also be particularly relevant to behaviours such as handwashing behaviour, where violations by others of such social contracts can eventually have hazardous outcomes for individuals. Therefore, despite a potential ambiguity in how social contracts of HCW handwashing behaviour may also apply to hazard management rules, overall it can be expected that it will have the same impact on patients' attention and memory.

Based on this above, an enhanced memory for the rarer behaviour in such contracts (e.g. breaking the contract) would be adaptive (Chang and Sanfey 2009). Furthermore, if individuals believe that the trustworthiness of certain occupations is a reliable predictor of future behaviour in social contracts, then this may well affect how accurately those professionals will be remembered. Accordingly, HCWs' hand hygiene could play an important role in patients' memory as such occupations can be considered high in trustworthiness. Hence, it is worth investigating if nurses and doctors would be better remembered when they did not wash their hands (low trustworthy behaviour) than when they did (high trustworthy behaviour), as the former would be considered a less frequent or unexpected behaviour (similar to Suzuki and Suga 2010). No significant differences are expected for individuals in lower trustworthy occupations in clinical settings (e.g. porters). In other words, a better understanding about how incongruence in occupations and behaviours affect memory may enable us to address the noted reluctance of patients to question HCWs about their hand-washing behaviour (McGuckin et al. 2001, 2004).

Here we investigated whether participants showed variations in response sensitivity $\left(d^{\prime}\right)$, biases $(C$.), and reaction time 
(RT) to faces of hospital staff linked to different hand-washing behaviours in different scenarios (Experiments 1 and 2). Experiment 3 investigated how different staff occupations affected face recognition. Furthermore, across the three experiments different forms of encoding were used (group, individual and par encoding) in order to examine different instances of real life situations in which people will need to encode faces (such as a face amongst a group of others, a face on its own, or a face compared with one other), such as would happen in a hospital setting. These different experimental paradigms test different memory loads during encoding, in order to investigate if variations of these affects information retrieval.

Overall it is hoped that an investigation into the adaptive processes that mediate memory and recognition of HCWs and their behaviour in handwashing contexts can inform future interventions and research. This is because it can shed light on what patients go through when faced with violations of this exchange, and may be used in the future to encourage reporting behaviour and/or prevention of cheating behaviour by HCWs, with the ultimate goal of increasing hand-washing behaviours in health-care settings.

\section{Method}

\section{Participants}

In Experiment 1, there were 32 participants (27 females; $M$ age $=22$ years, $S D=4$ ), 30 in Experiment 2 (26 females; $M$ age $=22$ years, $S D=4$ ), and 48 in Experiment 3 (42 females; $M$ age $=26$ years, $S D=6$ ). Although the participants were university students recruited via opportunity sampling, they were enrolled in one of UK's most ethnically and economically diverse student populations (http://www.kingston.ac.uk/ aboutkingstonuniversity/equality-diversity-and-inclusion/) representing over 100 countries. All participants had normal or corrected-to-normal vision.

Participants were informed that the experiment was about memory for faces, but no further details about the experiment were given.

\section{Materials}

Black and white photographs of the faces of 12 males and 12 females were selected from the Sheffield database (https:// www.sheffield.ac.uk/eee/research/iel/research/face). The frontal head-shot photographs $(126 \times 154$ pixels $)$ stood against a black background and had neutral expressions. Twelve faces ( $50 \%$ females) had to be memorized in groups of four faces (two males, two females), individually, or in pairs (one male, one female). The other 12 non-encoded faces were used in the subsequent recognition test. The experimental setup was similar to the one described in previous studies (Felisberti and Pavey 2010).

In the case of group encoding, the four photos were presented in one of four positions: upper left, upper right, lower left and lower right quadrants. Pairs of faces were presented on a horizontal plane, on the left and right side of a central fixation point. Individual images were presented on the centre of the screen. The viewing angle was $6 \times 5^{\circ}$ at approximately $60 \mathrm{~cm}$ from the centre of the monitor. E-Prime 2.0 (Psychology Software Tools, Pittsburgh, PA) was used to design and present the face recognition test.

\section{Procedure}

Hospital Scenario At the start of the test, participants read the following scenario on the screen (Fig. 1):

Jamie had a serious car accident and sustained a large gash to her leg that required 20 stitches. She had to stay in hospital to recover fully from her injuries. While there she observed the medical staff working in her ward. Next you will see slides with some of the medical staff. Before each slide there is a brief description of their hand-washing behavior. Your task is to memorise the faces and their behavior.

The screen following the scenario showed the behavioural descriptors, reporting either that the person/pair/ group (i) washed their hands after touching each of the patients in the ward (i.e. "clean hands"); (ii) never washed their hands after touching each of the patients in the ward (i.e. "dirty hands"); or (iii) only passed through the ward and did not touch the patients (i.e. "neutral"). The order of the presentation of the three groups was randomized and counterbalanced.

In Experiment 1, participants had to read a screen with a behavioural tag (unrestricted time), which was followed by a screen with a group of four faces. The duration of the face memorization was $6 \mathrm{~s}$ /group. The face encoding duration was short to examine recognition when the time for top-down cognitive processing was restricted.

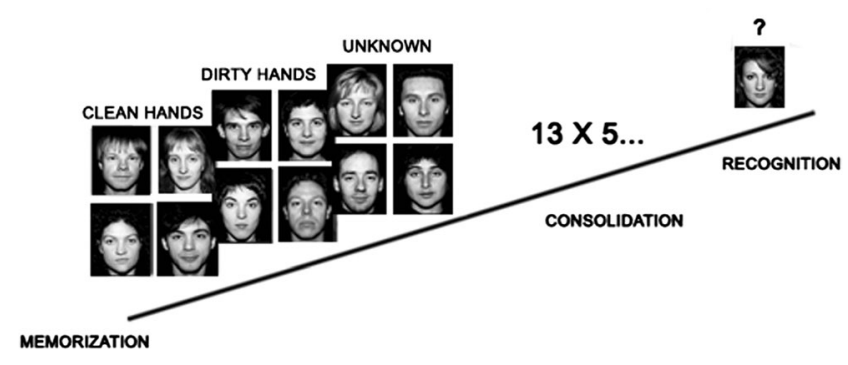

Fig. 1 Schematic representation of the core experimental setup 
In Experiment 2, the faces were presented individually and with their own behavioural descriptor. The encoding duration of each face was $10 \mathrm{~s}$ (which included the time to read the behavioural tags). The longer encoding duration than in the previous experiment aimed at increasing the time for topdown cognitive processing.

In Experiment 3, the behavioural descriptors included the staff occupations, which were either high trustworthy ('nurse') or low trustworthy ('porter'), otherwise they were similar to the ones in Experiments 1 and 2 . The descriptors were followed by a screen with pairs of faces. The duration of the presentation of each of pair of faces was $3 \mathrm{~s}$, an intermediate encoding duration in relation to previous experiments.

In all three experiments, the memorization task was followed by a simple multiplication task lasting 3-5 min. In the following recognition test, a face was presented (either an encoded or a new face) and participants had to answer if they had seen that face before ("old—yes"/"new—no").

\section{Data Analysis}

The participants' raw accuracy data was analysed in the framework of the signal detection theory (Green and Swets 1974; Macmillan 2002; Macmillan and Creelman 2004). The participants' ability to discriminate between 'signal' (i.e. encoded faces) and 'noise' (i.e. new faces) was estimated from the hit rate and the false alarm rate probabilities, which were converted into $z$-scores; the difference between $z$-transformed probabilities is the discriminability index or response sensitivity ( $d^{\prime}$ or $d$ prime), which in other words is a measure of the strength of the signal. An adjustment was used when the hit rate was $100 \%$ to avoid infinite $d^{\prime}$ values (Hautus 1995). The criterion $(C)$ is referred to as response bias, and it refers to the point where the noise and signal distributions cross over (i.e., $C=0$ and $\beta=1$ ). Negative values indicate a bias towards responding "yes" (i.e. liberal criterion), whereas positive values indicate a bias towards "no" (i.e. stricter criterion).

The repeated-measures ANCOVA had the behavioural descriptors tagged to faces as the within-participant factors (i.e. handwashing behaviour). The dependent variables were the $d$ ', response bias, and the RT. Due to the small number of males in the samples, the gender of the participants was always used as a covariate. All pair-wise comparisons were carried out using Bonferroni adjustments.

\section{Results}

\section{Experiment 1-Group Encoding}

This experiment aimed to test the effectiveness of brief encoding of groups of faces belonging to medical staff with different handwashing behaviours in the hypothetical hospital scenario, which were tagged to the encoded faces (dirty, clean, neutral hands/control). The encoding duration was $6 \mathrm{~s}$ per group of four faces and the time allocated to reading the behavioural tags was not restricted. Table 1 shows the mean, S.E., and $95 \%$ confidence interval for all three behaviours.

The $d^{\prime}$ to staff faces was significantly associated with their hand-washing behaviour $(F(2,60)=3.92, p=0.025$, $p \eta^{2}=0.12$; moderate effect size). Participants remembered the faces of medical staff with dirty hands better than the controls $(p=0.02)$. There was no difference in $d^{\prime}$ between staff with clean or dirty hands $(p=1.0)$, nor between clean hands and controls $(p=0.06)$.

The response bias $(C)$ to faces was also significantly associated with their hand-washing behaviour $(F(2,60)=4.02$, $p=0.02, p \eta^{2}=0.12$; moderate effect size). The $C$ for staff with clean and dirty hands was more relaxed than for controls ( $p=0.02$ and $p=0.01$, respectively). In other words, they tended to say "yes" to faces linked to clean and dirty hands, even when they might have been wrong, whereas this bias was either absent or inverse in the control/neutral group (Fig. 2a).

There were significant RT differences associated with staff hand-washing behaviours $(F(2,60)=5.04, p=0.009$, $\left.p \eta^{2}=0.14\right)$. The RT for clean hands tended to be faster than for dirty hands and controls, but no statistically reliable differences between the three groups were found (Fig. 2c).

\section{Experiment 2-Individual Encoding}

This experiment aimed at examining the effectiveness of individual face encoding with longer exposure for each face than in Experiment 1, which was brief ( $6 \mathrm{~s}$ for four faces). Although participants accuracy to the encoded faces in the previous experiment were relatively high (i.e. well above chance level), there was the possibility that the time to encode the faces linked to the behavioural descriptors was not long enough. Therefore, in this experiment the time to encode each face was increased to $10 \mathrm{~s}$.

The $d^{\prime}$ to faces of medical staff was not linked to their handwashing behaviour $\left(F(2,56)=1.08, p=0.35, p \eta^{2}=0.04\right)$ and nor was $C\left(F(2,56)=1.11, p=0.34, p \eta^{2}=0.04\right)$. Further studies are needed to clarify if the lack of associations was due to the higher response accuracy in comparison to the first experiment, since participants here had more time to encode the faces. As with the other variables, RT was not associated their hand-washing behaviours $(F(2,56)=2.03, p=0.14$, $p \eta^{2}=0.07$ ) (Fig. 2).

\section{Experiment 3-Pair Encoding and Occupation}

The aim here was to examine if the occupation of hospital staff interfered with the recognition of faces linked to different hand-washing behaviours. 
Table 1 The response sensitivity $\left(d^{\prime}\right)$, response bias $(C)$, and reaction time (RT) in Experiments 1-3

\begin{tabular}{llll}
\hline Group encoding $(N=32)$ & & $C$ & \\
\hline Hand-washing behaviour & $d^{\prime}$ & $-0.11(.07)[-0.25,0.03]$ & RT (ms) \\
\hline Clean & $1.94(.13)[1.67,2.20]$ & $-0.16(.07)[-0.31,-0.04]$ & $1421(82)[1253,1586]$ \\
Dirty & $2.02(.18)[1.66,2.39]$ & $0.10(.06)[-0.02,0.21]$ & $1569(94)[1378,1761]$ \\
Neutral & $1.55(.12)[1.31,1.80]$ & & $1527(102)[1318,1735]$ \\
Individual encoding $(N=32)$ & & $-0.09(.09)[-0.28,0.09]$ & $1169(92)[980,1358]$ \\
Clean & $2.73(.17)[2.37,3.08]$ & $-0.05(.09)[-0.22,0.13]$ & $1075(55)[964,1187]$ \\
Dirty & $2.66(.18)[2.28,3.03]$ & $0.10(.09)[-0.09,0.28]$ & $1102(62)[975,1230]$ \\
$\quad$ Neutral & $2.36(.16)[2.03,2.69]$ & & $1251(60)[1130,1373]$ \\
Occupation encoding $(N=48)$ & & $-0.09(.10)[-0.28,0.10]$ & $1375(80)[1214,1536]$ \\
Clean (nurses) & $2.36(.15)[2.06,2.66]$ & $-0.13(.11)[-0.34,0.08]$ & $1198(45)[106,1289]$ \\
Clean (porters) & $2.37(.16)[2.06,2.69]$ & $-0.15(.08)[-0.20,0.08]$ & $1362(61)[1238,1485]$ \\
Dirty (nurses) & $2.45(.18)[2.01,2.80]$ & $-0.05(.08)[-0.30,0.10]$ & $1258(61)[1135,1381]$ \\
Dirty (porters) & $2.35(.18)[1.99,2.71]$ & $-0.06(.07)[-0.21,0.09]$ & $1402(56)[1289,1515]$ \\
Neutral (nurses) & $2.23(.16)[1.91,2.56]$ & $-0.10(.09)[-0.27,0.08]$ &
\end{tabular}

The sample mean, standard error and the $95 \%$ confidence interval (in brackets) are given. Tagged hand-washing behaviours: dirty, clean, and neutral $N$ number of participants

The $d^{\prime}$ to the grouped faces of nurses and porters did not vary significantly with their hand-washing behaviour $(F<1)$. In line with $d^{\prime}$, $\mathrm{C}$ values also did not vary with porters and nurses hand-washing behaviour $(F<1)$.

No statistically reliable differences between the three handwashing conditions were observed ( $F<1$; Fig. 2 c). However, a pairwise comparison revealed significant RT differences associated with staff occupation in general $(p=0.002)$, whereby participants were faster at recognizing faces of nurses than porters across all handwashing conditions.

\section{Discussion}

Overall, these findings offered only partial support for an enhanced memory of faces linked to lack of hygiene in health settings. However, there were important and interesting findings across the three experiments worthy of further consideration.

When participants were asked to memorize the faces presented in groups and for a short duration there was increased recognition sensitivity to staff who did not wash their hands (Experiment 1). This suggests some evidence of an adaptive memory for violators (Cosmides and Tooby 1992). However this was only observed when compared to staff in a neutral setting (i.e. where the contract of hand-washing was not relevant). Further evidence of this was the response bias for groups of staff labelled as adhering to (washing hands) or violating (not washing hands) the social contract, compared to those whose behaviour was not relevant to the social contract (did not come into contact with patients). Neither of these effects were present when staff was presented individually in the memorization phase (Experiment 2). As there was more time for encoding in the second test, it may be that any effect of an adaptive memory for cheats or cooperators in social contracts only works as a heuristic when encoding time is limited. The occupation and hand-washing behaviour did not affect the participants' recognition of staff (Experiment 3). In other words, as opposed to previous research mentioned above, unexpected or incongruent information in relation to trustworthiness of staff (as presented from their occupation and behaviour) did not lead to an enhanced memory. Nonetheless, there was a faster RT to nurses than porters, albeit for all three hand-washing behaviours.

Do these results suggest that participants did not accurately perceive hand-washing and patient interaction as part of a social contract? This is unlikely, since it has been shown that an adaptive memory for cheats is found even with unfamiliar or artificial social contracts (Cosmides and Tooby 1992). However, what about the potential role of hazard management, as mentioned previously? Although it is argued that any such role in social contracts like handwashing would lead to the same behavioural outcomes, there may still be differences in terms of the strength of their relative effects. Farrelly and Turnbull (2008) found that overall, faces of individuals in social contracts overall were remembered better than those in hazard managements, even though cheaters were better remembered in both rules. Therefore, it is possible that the strength of purely hazard management rules on memory is 
a

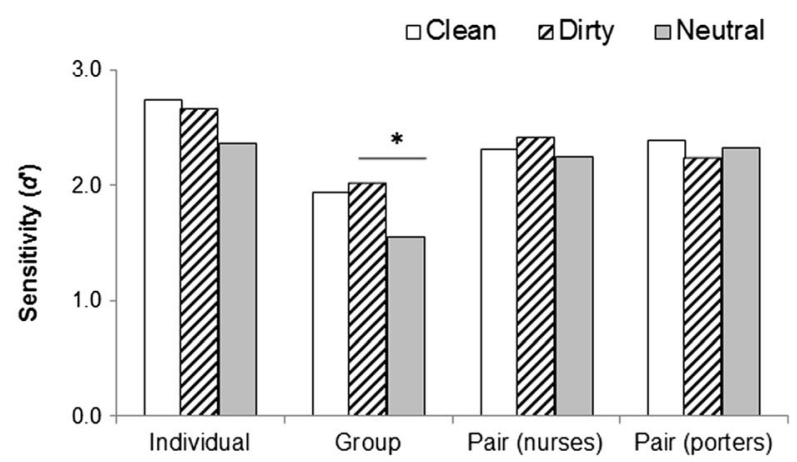

b

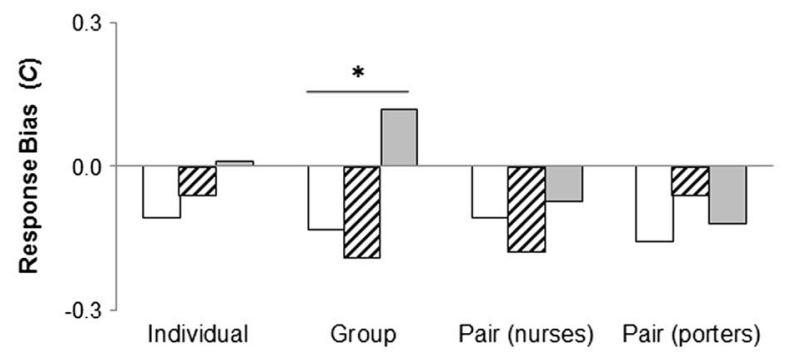

Encoding Type

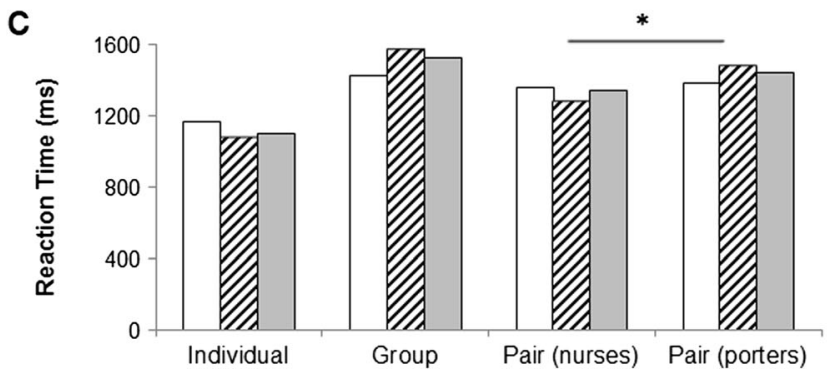

Fig. 2 The response sensitivity (a), response bias (b) and the reaction time (c) to faces of hospital staff tagged with different hand-washing behaviours (clean, dirty, neutral). There were three types of face encoding: "group encoding" (Expt. 1), "individual encoding" (Expt. 2), and "pair encoding" (Expt. 3)

less than for social contracts, and the lack of support for an enhanced memory of faces in these handwashing scenarios may suggest that participants were viewing these as purely hazard management scenarios and not social ones. This is unlikely, as argued previously many social contracts such as handwashing may also be hazardous for the health of others (for example, the social contract of not using a mobile phone while driving). Furthermore, the lack of clear differences between the memory of cheats and non-cheats in handwashing scenarios is contrary to what was found by Farrelly and Turnbull (2008) with purely hazard management rules (such as wearing a hard-hat on a building site). Overall, this suggests that any ambiguity in what type of rule or contract handwashing actually represents cannot account for the pattern of results observed in this research.
A further consideration is that although the role of porter was chosen for ecological validity (i.e. common occupation in clinical settings where hand-washing is important, but not primary HCWs), they may well be viewed as trustworthy as nurses, and as playing an important role in patient care and well-being. However, nurses were recognized faster overall than porters, which suggests occupations did influence how participants processed the faces of individuals in the study. This may be due to alternative explanations other than trustworthiness, such as differences in perceived status. Finally, another possible reason for the lack of significant findings in these experiments is that the effect was not strong enough in artificial laboratory settings with non-clinical participants. Hence, one clearly important advancement of this research is to involve real patients as participants in an actual clinical setting, where hand-washing behaviour is an important and pertinent aspect for their time in hospital.

The present study has benefits to add to the existing literature on the patient's role in promoting hand-washing behaviour in HCWs by examining actual behaviour, rather than intentions, which is the case with the majority of existing studies. The absence of a strong memory advantage towards specific behaviours suggests that, in addition to reminders about the importance of washing one's hands, we need to devise strategies to remind people of the importance to observe (and accurately remember) the hand-washing behaviour of others in health environments. The current experiments introduce a novel approach to this, and future research highlighted above can offer a valuable means by which this can be better understood, and eventually shape successful interventions in clinical settings so that hand-washing by all is increased.

Acknowledgments We would like to thank the research assistant TorArne Haugland for most of the data collection. This study was partially supported by the Staff Research Capability Fund from Kingston University London to FMF.

Compliance with Ethical Standards The University Ethics Committee approved these experiments, which were conducted according to the ethical standards of the Declaration of Helsinki 1964.

Open Access This article is distributed under the terms of the Creative Commons Attribution 4.0 International License (http:// creativecommons.org/licenses/by/4.0/), which permits unrestricted use, distribution, and reproduction in any medium, provided you give appropriate credit to the original author(s) and the source, provide a link to the Creative Commons license, and indicate if changes were made.

\section{References}

Barclay, P. (2008). Enhanced recognition of defectors depends on their rarity. Cognition, 107, 817-828.

Barclay, P., \& Lalumière, M. L. (2006). Do people differentially remember cheaters? Evolution and Human Behavior, 17, 98-113. 
Bell, R., \& Buchner, A. (2012). How adaptive is memory for cheaters? Current Directions in Psychological Science, 21, 403-408.

Bell, R., Buchner, A., Kroneisen, M., \& Giang, T. (2012). On the flexibility of social source memory: a test of the emotional incongruity hypothesis. Journal of Experimental Psychology. Learning, Memory, and Cognition, 38, 1512-1529.

Chang, L. J., \& Sanfey, A. G. (2009). Unforgettable ultimatums? Expectation violations promote enhanced social memory following economic bargaining. Frontiers in Behavioral Neuroscience, 3, 36.

Cosmides, L. (1989). The logic of social exchange: has natural selection shaped how humans reason? Studies with the Wason selection task. Cognition, 31, 187-276.

Cosmides, L., \& Tooby, J. (1992). Cognitive adaptations for social exchange. In J. Barkow, L. Cosmides, \& J. Tooby (Eds.), The adapted mind (pp. 163-228). New York: Oxford University Press.

Davis, R., Parand, A., Pinto, A., \& Buetow, S. (2015). Systematic review of the effectiveness of strategies to encourage patients to remind healthcare professionals about their hand hygiene. Journal of Hospital Infection, 89, 141-162.

Ermer, E., Guerin, S. A., Cosmides, L., Tooby, J., \& Miller, M. B. (2006). Theory of mind broad and narrow: reasoning about social exchange engages ToM areas, precautionary reasoning does not. Social Neuroscience, 1, 196-219.

Farrelly, D., \& Turnbull, N. (2008). The role of reasoning domain on face recognition: detecting violations of social contract and hazard management rules. Evolutionary Psychology, 6, 523-537.

Felisberti, F. M., \& Pavey, L. (2010). Contextual modulation of biases in face recognition. PLOS ONE, 5, e12939.

Fiddick, L. (2004). Domains of deontic reasoning: resolving the discrepancy between the cognitive and moral reasoning literatures. The Quarterly Journal of Experimental Psychology. A Human Experimental Psychology, 57, 447-74.

Fiddick, L., Cosmides, L., \& Tooby, J. (2000). No interpretation without representation: the role of domain-specific representations and inferences in the Wason selection task. Cognition, 77, 1-79.

Fiddick, L., Spampinato, M. V., \& Grafman, J. (2005). Social contracts and precautions activate different neurological systems: an fMRI investigation of deontic reasoning. Neurolmage, 28, 778-786.

Green, D. M., \& Swets, J. A. (1974). Signal detection theory and psychophysics. New York: Krieger.

Hautus, M. J. (1995). Corrections for extreme proportions and their biasing effects on estimated values of d'. Behavior Research Methods, Instruments, \& Computers, 27, 46-51.

Kroneisen, M., \& Bell, R. (2012). Sex, cheating, and disgust: enhanced source memory for trait information that violates gender stereotypes. Memory, 21, 1-15.

Lent, V., Eckstein, E. C., Cameron, A. S., Budavich, R., Eckstein, B. C., \& Donskey, C. J. (2009). Evaluation of patient participation in a patient empowerment initiative to improve hand hygiene practices in a Veterans Affairs medical center. American Journal of Infection Control, 37, 117-120.
Longtin, Y., Sax, H., Allegranzi, B., Schneider, F., \& Pittet, D. (2011). Hand hygiene. New England Journal of Medicine, 364, e24.

Macmillan, N.A. (2002). Signal detection theory. In H. Pashler and J. Wixted (Eds.),: Stevens' Handbook of Experimental Psychology, 3rd edition. Volume 4: Methodology in Experimental Psychology (pp.43-67). John Wiley \& Sons, Inc.

Macmillan, N. A., \& Creelman, C. D. (2004). Detection theory: a user's guide. Mahwah: Lawrence Erlbaum Associates.

McGuckin, M., Waterman, R., Storr, J., Bowler, I. C. J. W., Ashby, M., Topley, K., \& Porten, L. (2001). Evaluation of a patient-empowering hand hygiene programme in the UK. Journal of Hospital Infection, 48, 222-227.

McGuckin, M., Taylor, A., Martin, V., Porten, L., \& Salcido, R. (2004). Evaluation of a patient education model for increasing hand hygiene compliance in an inpatient rehabilitation unit. American Journal of Infection Control, 32, 235-238.

Mealey, L., Daood, C., \& Krage, M. (1996). Enhanced memory for faces of cheaters. Evolution and Human Behavior, 17, 119-128.

Oda, R. (1997). Biased face recognition in the Prisoner's Dilemma game. Evolution and Human Behavior, 18, 309-315.

Oda, R., \& Nakajima, S. (2010). Biased face recognition in the Faith Game. Evolution and Human Behavior, 31, 118-122.

Pittet, D., Dharan, S., Touveneau, S., Sauvan, V., \& Perneger, T. V. (1999). Bacterial contamination of the hands of hospital staff during routine patient care. Archive of Internal Medicine, 159, 821-826.

Salmon, S., Pittet, D., Sax, H., \& McLaws, M. L. (2015). The "My five moments for hand hygiene" concept for the overcrowded setting in resource-limited healthcare systems. Journal of Hospital Infection, 91, 95-99.

Sladek, R. M., Bond, M. J., \& Phillips, P. A. (2008). Why don’t doctors wash their hands? A correlational study of thinking styles and hand hygiene. American Journal of Infection Control, 36, 399-406.

Stone, V. E., Cosmides, L., Tooby, J., Kroll, N., \& Knight, R. T. (2002). Selective impairment of reasoning about social exchange in a patient with bilateral limbic system damage. Proceedings of the National Academy of Sciences of the United States of America, 99, 11531-11536.

Sugiyama, L., Tooby, J., \& Cosmides, L. (2002). Cross-cultural evidence of cognitive adaptations for social exchange among the Shiwiar of Ecuadorian Amazonia. Proceedings of the National Academy of Sciences, 99, 11537-11542.

Suzuki, A., \& Suga, S. (2010). Enhanced memory for the wolf in sheep's clothing: facial trustworthiness modulates face-trait associative memory. Cognition, 117, 224-229.

Volstorf, J., Rieskamp, J., \& Stevens, J. R. (2011). The good, the bad, and the rare: memory for partners in social interactions. PLoS ONE, 6, e18945.

Wason, P. (1966). Reasoning. In B. M. Foss (Ed.), New horizons in psychology. Harmondsworth: Penguin.

WHO. (2009). World Health Organization guidelines for hand hygiene in health care. Geneva: World Health Organization. 